\title{
Providing Service Differentiation for Optical-Burst-Switched Networks
}

\author{
Wanjiun Liao, Member, IEEE, and Chi-Hong Loi
}

\begin{abstract}
This paper proposes a service differentiation scheme called preemptive wavelength reservation protocol (PWRP) to provide proportional quality of service $(\mathrm{QoS})$ for optical-burst-switched (OBS) networks. In the context of service differentiation, traffic is divided into different service classes based on traffic characteristics. A service differentiation scheme then provides different degrees of resource assurance to different classes of traffic in proportion to their service classes. Unlike existing approaches, which may degrade to classless schemes or which may suffer from low wavelength utilization, the mechanism in this paper is robust and efficient and supports an incremental deployment of QoS support. A usage profile for each class is maintained at the router, and a preemptive wavelength reservation mechanism is implemented to ensure QoS. An analytical model is derived and simulations are conducted to evaluate the performance. The results show that the approach described in this paper performs better than existing mechanisms in terms of lower blocking probability and higher resource utilization, making it an excellent QoS mechanism for OBS networks.
\end{abstract}

Index Terms-Optical burst switching, preemptive wavelength reservation protocol (PWRP), service differentiation, wavelength reservation.

\section{INTRODUCTION}

$\mathbf{O}$ PTICAL burst switching [1], [2] is a promising solution to providing terabit optical routing and building an all-optical wavelength-division-multiplexing (WDM) layer for the optical Internet. Based on the concept of "burst" switching, optical burst switching groups several Internet protocol (IP) packets with the same network egress address and common attributes like quality of services (QoS) into a burst and then forwards the burst through the network as a single entity. Each burst consists of a control header and a burst payload. The data burst (i.e., payload) and the header are transmitted on separate wavelengths (or channels), with the burst header transmitted slightly ahead of time. This allows optical core routers to process the headers electronically in advance for establishment of a virtual end-to-end optical path and then to switch data bursts optically. Based on a one-way reservation protocol, optical burst

Manuscript received October 7, 2003; revised April 20, 2004. This work was supported in part by the Ministry of Education (MOE) program for Promoting Academic Excellence of Universities under Grant 89E-FA06-2-4-7 and in part by the National Science Council, Taiwan , R.O.C., under Grant NSC932213-E-002-001.

W. Liao is with the Department of Electrical Engineering and the Graduate Institute of Communications Engineering, National Taiwan University, Taipei, Taiwan 106, R.O.C. (e-mail: wjliao@ntu.edu.tw; wjliao@cc.ee.ntu.edu.tw).

C.-H. Loi was with the Department of Electrical Engineering and the Graduate Institute of Communications Engineering, National Taiwan University, Taipei, Taiwan 106, R.O.C. He is now with SENAO International Company, Taiwan, R.O.C.

Digital Object Identifier 10.1109/JLT.2004.831150 switching precedes a data burst with a control header in a predefined offset time, without waiting for an acknowledgment before the beginning of the data transmission.

Current IP provides only best-effort service to deliver variable-length packets. The future Internet may demand differentiated service for multimedia applications. In the context of service differentiation, traffic is divided into different service classes based on traffic characteristics. A service differentiation scheme provides different degrees of resource assurance to different classes of traffic in proportion to their service classes. For the optical Internet to be truly ubiquitous, one must address, among other important issues, how the WDM layer supports differentiated service. There are many mechanisms to implement connectionless QoS in the literature [3]-[5], mostly using buffers and scheduling. These approaches, however, incur high processing overhead at intermediate nodes and mandate a certain amount of buffers for switching. To date, no efficient optical buffer is available. The use of electronic buffers necessitates optical-electrical-optical (O/E/O) conversions, which must be avoided in an all-optical network where data is kept in the optical domain at all intermediate nodes. The other alternative is the use of optical fiber-delay lines (FDLs), which provide very limited delay functionality at optical-burst-switched (OBS) switches. This requires new QoS mechanisms that do not require buffers at the WDM layer in OBS networks.

In [6], a new approach is proposed in which different offset time is assigned to different service classes over just-enough-time (JET) [2] OBS networks so as to provide differentiated services in terms of burst loss probability for classes of different priorities (termed JET QoS in the remainder of the paper). JET QoS, however, has the following problems. First, it requires a homogeneous reservation mechanism, in which the burst length and the offset time of each class should be set and kept at the same value at all routers in an OBS domain; otherwise, it may degrade to a classless approach like the original JET. The offset time, however, may not be maintainable at the routers due to possibly longer rerouting paths and congestion on the control channels, rendering the mechanism unable to provide QoS. Second, JET QoS reduces the loss rates of high-priority traffic at the expense of an increase in the loss rates of lower priority traffic. However, it does not limit how much resource each class of bursts can use in a given period. As a result, only simple service differentiation can be provided, but not proportional differentiated service. Worse, JET QoS may starve lower priority bursts because higher priority bursts always have more opportunities to make wavelength reservation. In [7], the authors point out that this treatment is particularly unfair to long bursts of low priority 
because it is hard to find a long gap to serve a long burst of low priority in an almost full schedule table. In order to cope with the unfairness, a proportional QoS scheme is studied in [7]. The loss rate of each class is maintained in a predefined proportion according to the priority. A packet will be dropped if its predefined loss rate is violated, regardless of whether there is an idle channel. The intentional dropping gives more and longer free periods of wavelengths on the output link to admit high-priority bursts. This approach, however, causes excessive dropping. As a result, it has higher overall blocking probability and worse wavelength utilization compared with classless approaches like JET. Moreover, in [7], there is no study on the setting of the proportion factor. Improper setting may lead to high blocking probabilities of lower priority classes and the inability to enforce service differentiation for different classes of traffic. Other service differentiation schemes in OBS are described in [8]-[10]. In [8], a proportional bandwidth scheme is used in parallel with policing on the burst assembly mechanism and with FDL buffering. In [9], a prioritized burst segmentation technique is used to resolve contention in the optical core. A burst deflection and composition burst aggregation strategy is also employed. In [10], a two-way reservation lightpath reservation, along with a centralized scheduling technique, is used to provide bounded blocking probabilities on a burst-by-burst basis.

In this paper, the preemptive wavelength reservation protocol (PWRP) is proposed to provide service differentiation for OBS networks without requiring buffers at the WDM layer. We maintain a usage profile for each class at the router and implement a preemptive wavelength reservation algorithm to ensure QoS. Unlike JET QoS, which is unfair and may degrade to a classless mechanism, PWRP is robust under network congestion, supports incremental QoS deployment, and cooperates well with any "best-effort" reservation mechanisms like Horizon [1] and the original JET [2]. Furthermore, our protocol provides service differentiation without the excessive dropping problem suffered by [7], thus enjoying high wavelength utilization. Here, an analytical model is derived and simulations conducted to evaluate the performance of the proposed mechanism. The results show that PWRP performs better than JET QoS [6] and Drop [7] in terms of lower blocking probability and higher resource utilization, making it an excellent QoS mechanism for OBS networks.

The remainder of the paper is organized as follows. Section II describes thePWRP for OBS networks. Section III presents an analytical model and the simulation results to evaluate the performance of PWRP. Finally, concluding remarks are included in Section IV.

\section{Preemptive Wavelength Reservation Protocol}

In this section, we describe the proposed preemptive wavelength reservation mechanism for the OBS network.

\section{A. Protocol Fundamentals}

Suppose that each switch has a total of $m$ wavelengths per output link to serve data bursts. Considering the characteristics of traffic, we classify bursts into $k$ different classes of service, say $c_{1}, c_{2}, \ldots c_{k}$, and assign each class a service priority.
Without loss of generality, classes $c_{1}, c_{2}, \ldots c_{k}$ are assumed to have priority in an ascending order. The higher priority has the lower blocking probability. Since the blocking probability at a switch is inversely proportional to the number of idle wavelengths, we let classes of higher priorities use more resources (i.e., wavelengths). In other words, if class $c_{j}$ has priority over class $c_{i}$, class $c_{j}$ bursts are allowed to use more resources than class $c_{i}$. Each class is associated with a usage limit, defined as a fraction of the system resources the class is allowed to use. Let $p_{i}$ be the usage limit assigned to class $c_{i} .0 \leq p_{1}<p_{2}<\cdots<$ $p_{k} \leq 1$, and $\sum_{i=1}^{k} p_{i}=1$.

In our protocol, each class is associated with a predefined usage limit. Each switch maintains a usage profile for a class per output link and monitors the current usage of each class. Based on the profile table, the switch can determine if there is an eligible wavelength on the outgoing link to schedule a new request. A wavelength is eligible to a request, say $R$, if it is not assigned to any other request during the burst duration requested by $R$.

The usage profile of each class records a predefined usage limit, a current usage, and a list of scheduled requests in the same class with the following triple: burst duration, outgoing wavelength, and a predefined timer. The burst duration can be calculated according to the burst header, which carries an offset time (i.e., the time difference between the control header and the data burst) and a burst length. Let $l, s$, and $e$ be the burst length, the start time, and the end time of a data burst, respectively. The start time $s$ is equal to the current time plus the offset time carried by the control header. Clearly, $e=s+l$. The burst duration is maintained in the format of start time/end time of the data burst. The outgoing wavelength may be an eligible wavelength or a preempted wavelength scheduled to transmit the data burst in the burst duration. The predefined timer records the maximum tolerable waiting time for the receipt of the data burst, in an attempt to cope with network faults and the preemption incurred by our protocol.

\section{B. In-Profile Verification}

A class of traffic is said to be in profile if its current usage does not exceed a predefined usage limit; otherwise, the class is out of profile. Intuitively, the usage of class $c_{i}$ is defined as the total duration of the scheduled bursts of class $c_{i}$ over the total duration of the scheduled bursts of all classes. Considering the bursty nature of traffic, the class usage is monitored in a short time period, say $\tau$, which is called the monitoring time scale in [7]. Let $\rho_{i}$ denote the current usage of class $c_{i}$, defined as follows: $\rho_{i}=\sum_{j=1}^{n_{i}} l_{j} / \sum_{i=1}^{k} \sum_{j=1}^{n_{i}} l_{j}$, where $k$ is the number of classes, $n_{j}$ is the number of scheduled requests of class $c_{j}$ in $(t, t+\tau)$, and $l_{j}$ is the burst length of request $R_{j}$ in class $c_{j}$.

Class $c_{i}$ is said to be in profile if $\rho_{i} \leq p_{i}$; otherwise, the class is out of profile.

\section{Burst Preemption}

Suppose that a switch has just received $R_{x}$, an in-profile class request, with start time and end time of $s_{x}$ and $e_{x}$, respectively. Let $C_{O}$ be a set of candidate classes in which a wavelength is to be preempted, defined as 
1) $C_{O}=\left\{c_{i} \mid \rho_{i}>p_{i}, i=1,2, \ldots k\right\}$

2) $\forall c_{i} \in C_{O}, \exists R_{v} \in c_{i}, s_{v}-\varepsilon_{\mathrm{s}} \leq s_{x} \leq e_{x} \leq e_{v}+\varepsilon_{e}$

where $\rho_{i}$ and $p_{i}$ are the current usage and the usage limit of class $c_{i}$, respectively; $\varepsilon_{s}$ and $\varepsilon_{e}$ are the gaps to the previous and next scheduled requests, respectively, on the same wavelength as request $R_{v}$, a scheduled out-of-profile request; and $s_{v}$ and $e_{v}$ are the start time and end time of request $R_{v}$, respectively. These two conditions imply that every class in $C_{O}$ must be out-of-profile (by condition (1)) and must at least include a request previously scheduled, and its burst duration plus the two gaps to the previous and next scheduled requests on the same wavelength can cover the newly received request (by condition (2)).

The preemption process is triggered when there is no eligible wavelength on the outgoing link to schedule the in-profile request $R_{x}$. The process proceeds as follows. The switch preempts a wavelength from a scheduled out-of-profile request in the set of candidate classes $C_{O}$, starting from the class with the lowest priority to the highest priority. Request $R_{p}$ will be the victim to be preempted if the preempted wavelength is eligible to serve request $R_{x}$ (i.e., the burst duration of request $R_{p}$, plus the two gaps to the previous and the next requests, are eligible to request $R_{x}$ ). In other words, the time period of a wavelength can be used to schedule $R_{x}$ if $R_{p}$ is preempted.

\section{Operation Overview}

A switch keeps monitoring its usage table. Upon receiving a class $c_{i}$ request, the switch first attempts to identify a wavelength eligible to the request. If the attempt succeeds, the request is scheduled, and the usage profile of class $c_{i}$ is updated; otherwise, the following takes place. The switch examines if the class to which the request belongs is in profile, using the in-profile verification algorithm described in Section II-B. If it is in profile, a previous scheduled request of an "out-of-profile" class is preempted using the burst preemption algorithm described in Section II-C; otherwise, the request is rejected, and the data burst is simply dropped. Once a wavelength is preempted, the switch updates the current usage of both requests' classes accordingly. Fig. 1 summarizes the operation of the PWRP.

A switch may schedule a request either with an eligible wavelength or a preempted wavelength. To prevent a scheduled request from being preempted at any switch in the data path, thereby wasting resources due to inconsistent scheduling, a switch should be able to detect preemption to previously scheduled bursts by any switch. We discuss this issue for two cases.

1) Partial preemption: In this case, a burst is preempted during the transmission. The switch truncates the burst and sends out a signal to end the burst. Thus, all switches in the channel learn about the preemption.

2) Full preemption: In this case, a burst is preempted before the start of the transmission. Recall that each pair (burst duration/outgoing wavelength) is associated with a predefined timer in the usage profile. The timer is activated either at the requested start time of a burst or in the middle of any burst transmission when a data packet is not received in time. On expiry of the timer, the switch

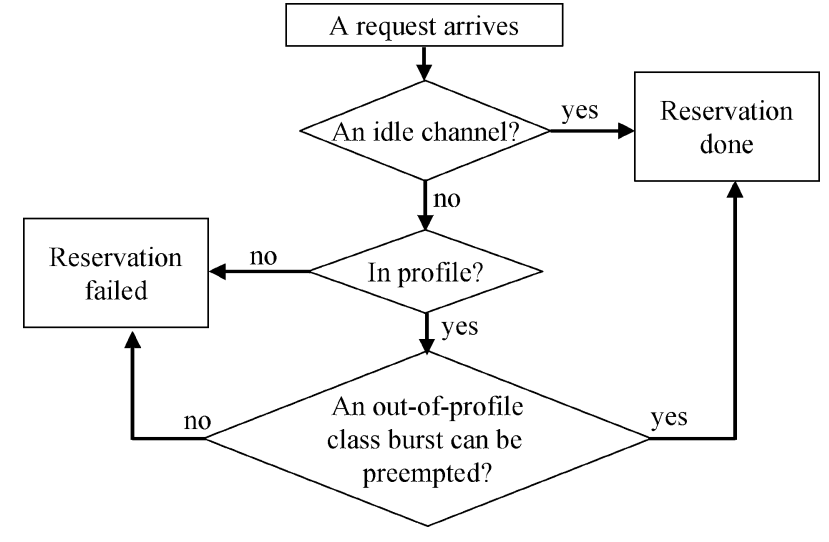

Fig. 1. Simplified flowchart of the operation.

assumes an occurrence of a fault (either a physical fault or a preemption) if no data burst has been received.

In either case (i.e., time-out or on receipt of a preemption signal), the switch will remove the switching information for the associated burst and make the wavelength available for other requests.

\section{E. Example}

Figs. 2 and 3 show two examples to demonstrate how the preemptive mechanism works. Suppose that there are four wavelengths on each link at a switch, and two classes of service are supported in the system. The shaded bars (i.e., bursts 1, 2, and 3) indicate class 1 , and the white bars (i.e., burst 4) represent class 2. Class 2 traffic is assumed to have priority over class 1. Fig. 2(a) shows the snapshot of a wavelength reservation schedule at a switch when a new burst header of class 2 traffic arrives. In this example, class 1 traffic is assumed to be out of profile. Fig. 2(b) shows that the new arrival request of class 2 finds no idle channel available at the burst header arrival time. Since class 1 is out of profile, a victim should be preempted to release the resource for this new burst. Suppose the data burst that will start last is selected to be preempted. ${ }^{1}$ Thus, burst 1 is preempted, and wavelength $\lambda 1$ is released as shown in Fig. 2(c). The preempted wavelength $\lambda 1$ is used to schedule the new burst, as shown in Fig. 2(d).

Each scheduled burst is associated with a predefined time, say, $\xi$. For example, burst 4 is expected to arrive on $\lambda 4$ at time $t_{s}$ in Fig. 2(a). If the data burst does not arrive between time $t_{s}$ and $t_{s}+\xi$, the resource is released. This mechanism prevents resources to be reserved by bursts either preempted by previous routers or dropped due to other network faults.

Fig. 3 shows an example of partial preemption. In this example, burst 2 is the victim to be preempted. But since the data burst of burst 2 will arrive at this switch earlier than the new request, only a portion of burst 2 will be preempted. Thus, wavelength $\lambda 2$ is rescheduled for the new request as shown in Fig. 3(d). When burst 2 is truncated due to the preemption, the switch has to send out a signal to end the transmission.

\footnotetext{
${ }^{1}$ Note that in our mechanism, we do not define how to select a victim in a class to be preempted. In our simulation, the data burst that will start last is preempted, as in this example.
} 


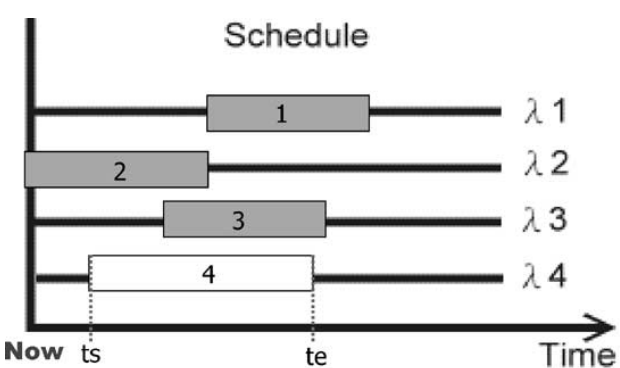

(a)

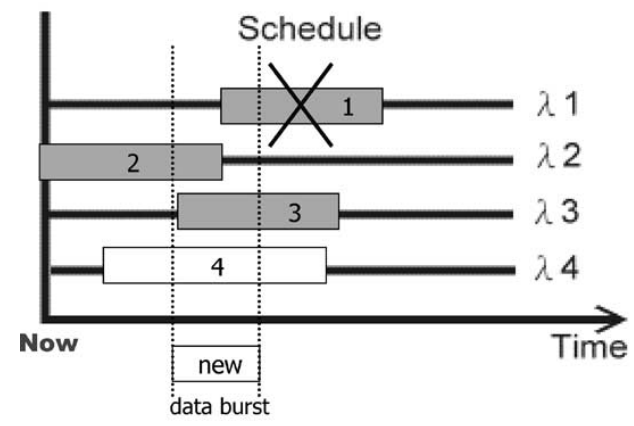

(c)

Fig. 2. Example of full preemption.

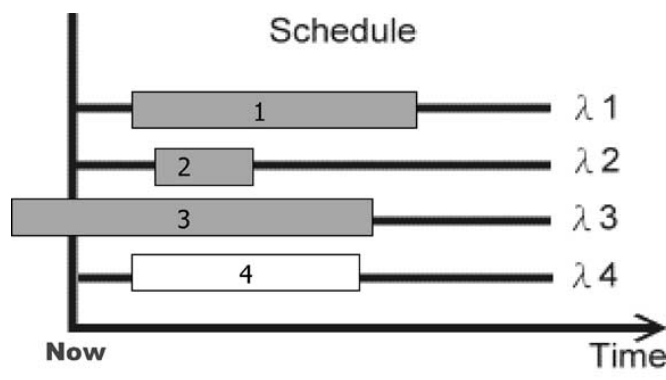

(a)

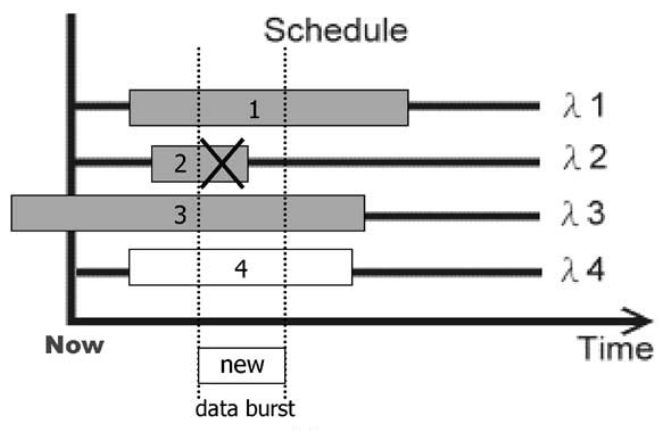

(c)

Fig. 3. Example of partial preemption.

\section{Performance Evaluation}

\section{A. Mathematical Analysis}

In this section, we analyze the performance of the proposed preemptive reservation mechanism. To simplify the analysis, we assume that both burst length and interarrival times are exponentially distributed with an average of $L$. The offset time between

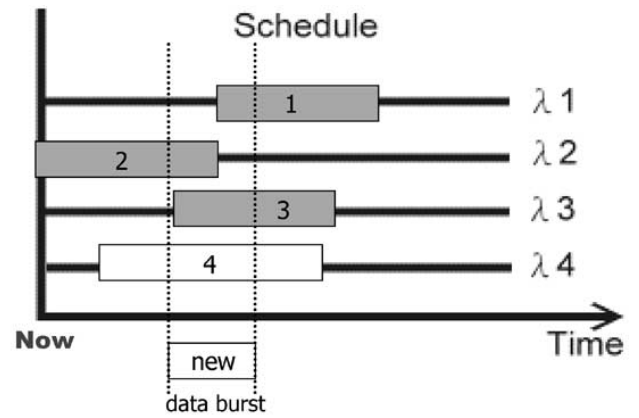

(b)

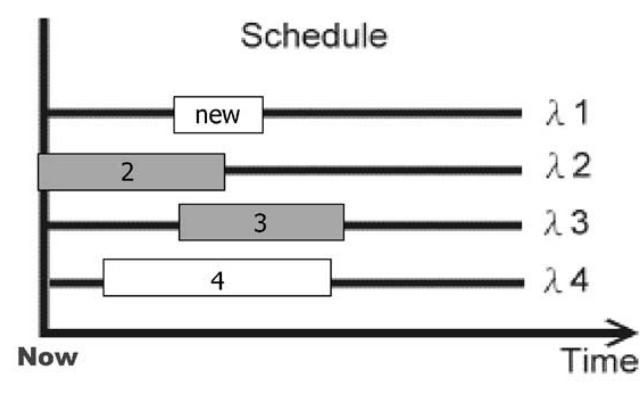

(d)

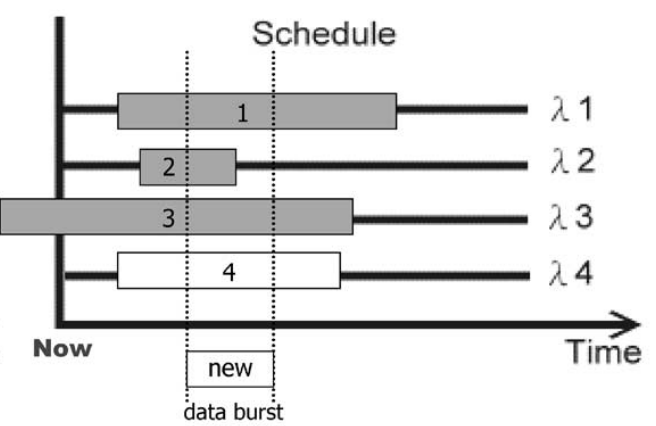

(b)

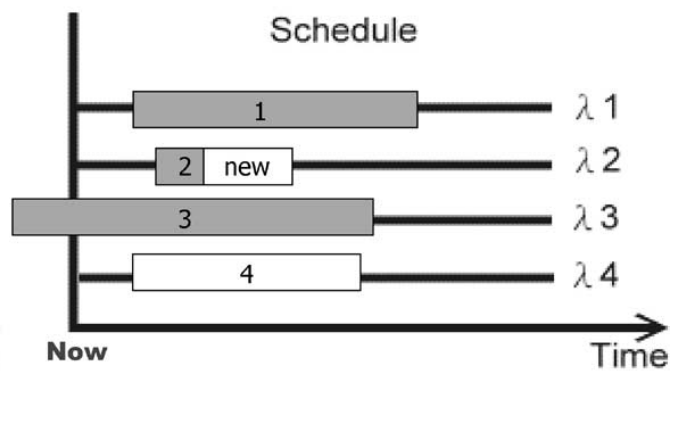

(d)

the burst header and payload is assumed constant. We consider $K$ classes of services in the system and $m$ wavelengths on each link of a switch.

We model the number of wavelengths used by each class at a switch as a continuous-time Markov chain. Let $\lambda_{i}$ and $\mu_{i}$ be the arrival rate and the service rate of class $c_{i}$, respectively. Let $\bar{n}=$ $\left(n_{1}, n_{2}, \ldots, n_{k}\right)$ denote the state of the Markov chain, where $n_{i}$ 
is the number of wavelengths used by class $c_{i}$ (i.e., the current usage of class $c_{i}$ is $\rho_{i}=n_{i} / m$ ). In our analysis, we assume $\tau$ is equal to 0 . In other words, whether a request is accepted depends on the state at the moment that the request arrives plus the offset time. The state space of the Markov chain can be derived as $S=\left\{\bar{n} \mid \sum_{i=1}^{K} n_{i} \leq m, 0 \leq n_{i} \leq m, 1 \leq i \leq K\right\}$

Let $P_{\bar{n}, \bar{n}\left(j^{+}\right)}, P_{\bar{n}, \bar{n}\left(j^{-}\right)}$, and $P_{\bar{n}, \bar{n}\left(i^{+}, j^{-}\right)}$be the transition rate from $\bar{n}$ to $\bar{n}\left(j^{+}\right), \bar{n}\left(j^{-}\right)$, and $\bar{n}\left(i^{+}, j^{-}\right)$, where $\bar{n}\left(j^{+}\right)=\left(n_{1}, \ldots, n_{j-1}, n_{j}+1, n_{j+1}, \ldots, n_{k}\right)$, $\bar{n}\left(j^{-}\right)=\left(n_{1}, \ldots, n_{j-1}, n_{j}-1, n_{j+1}, \ldots, n_{k}\right)$, and the first equation shown at the bottom of the page. Subject to the limited number of outgoing wavelengths, the transition rate can be described in the following two cases:

1) Case 1: when $\sum_{i=1}^{K} n_{i}<m$

$$
\begin{aligned}
& P_{\bar{n}, \bar{n}^{\left(j^{+}\right)}}=\lambda_{j} \\
& P_{\bar{n}, \bar{n}\left(j^{-}\right)}= \begin{cases}n_{j} \mu_{j}, & \text { if } n_{j}>0 \\
0, & \text { otherwise }\end{cases}
\end{aligned}
$$

2) Case 2: when $\sum_{i=1}^{K} n_{i}=m$

$$
\begin{aligned}
P_{\bar{n}, \bar{n}\left(i^{+}, j^{-}\right)} & = \begin{cases}\lambda_{i}, & \text { if } n_{i}<r_{i}, j=\min \left\{h \mid n_{h}>r_{h}\right\} \\
0, & \text { otherwise }\end{cases} \\
P_{\bar{n}, \bar{n}\left(j^{-}\right)} & = \begin{cases}n_{j} \mu_{j}, & \text { if } n_{j}>0 \\
0, & \text { otherwise }\end{cases}
\end{aligned}
$$

where $r_{i}$ denotes the number of wavelengths class $c_{i}$ is allowed to use (i.e., the usage limit of class $c_{i}$ is $p_{i}=r_{i} / m$ ). Since the Markov chain is aperiodic, finite, and irreducible, the stationary probability $p(\bar{n})$ exists. From the stationary probability, we can derive the following performance matrix:

1) The blocking probability of a new arrival burst of class $c_{i}$ is given by

$$
\left.P_{\text {block }}(i)=\sum_{\bar{n} \in\left\{\left(n_{1}, \ldots, n_{k}\right) \mid \sum_{j=1}^{K} n_{j}=m, n_{i} \geq r_{i}\right.}\right\} .
$$

2) The preempted probability of an accepted burst of class $c_{i}$ is given by the last equation shown at the bottom of the page, where $N_{\text {preempted }}(i)$ is the number of class $c_{i}$ bursts preempted, and $N_{\text {accepted }}(i)$ is the number of class $c_{i}$ bursts accepted.

3) The total blocking probability of a burst is defined as $P_{\text {total_block }}(i)=P_{\text {new }}(i)+P_{\text {preempted }}(i)$.

4) The utilization is defined as $U=\sum_{\bar{n} \in S} \sum_{i=1}^{K} n_{i}$ $\times p(\bar{n})$.

5) The proportion of bandwidth used by class $i$ is defined as $U_{i}=\left(\sum_{n \in S} n_{i} \times p(\bar{n})\right) / U$.

Our analytical results are validated by simulation in Section III-C.

\section{B. Simulation}

In this section, the performance of PWRP is compared with classless (i.e., best effort) JET QoS [6] and Drop [7] by simulation. The classless mechanism may be Horizon [1] or the original JET [2]. The JET QoS is the original JET with different extra offset times assigned to different classes of bursts. Drop refers to the intentional dropping approach proposed in [7].

We consider bufferless switches with $m=8$ wavelengths per output link. Each switch is assumed to be capable of full wavelength conversion (i.e., a wavelength can be converted to any other wavelength). The numbers of input/output ports per OBS switch are all eight. The average burst length is $100 \mathrm{kB}$, and the wavelength bit rate is $10 \mathrm{~Gb} / \mathrm{s}$. We assume there are $k$ classes, all of which generate bursts with an exponential interarrival time and exponential burst duration. To simplify the computation and without loss of generality, the simulation is based on the assumption that all sources have the same arrival rates (i.e., $\lambda_{1}=\lambda_{2}=\cdots=\lambda_{k}=\lambda$ ) and service rates (i.e., $\mu_{1}=\mu_{2}=\cdots=\mu_{k}=\mu$ ).

In the first experiment, we observe the behavior of the four approaches at a single bufferless OBS switch. We consider two classes only, i.e., classes 1 and 2. We let class 2 have priority over class 1 , and assign the usage limits of 0.0 and 1.0 to classes 1 and 2, respectively. Thus, class 2's traffic can preempt class 1's traffic when necessary. Fig. 4 shows the blocking probability of each class for the four approaches, varying the offered load from 0.4 to 1 . The offered load here is defined as $\sum_{i} \lambda_{i} / m \mu$, where $m$ is the number of wavelengths on each link, $\lambda_{i}$ is the burst arrival rate of class $c_{i}$, and $\mu$ is the service rate of each burst, which is equal to the average burst length divided by the wavelength bit

$$
\bar{n}\left(i^{+}, j^{-}\right)= \begin{cases}\left(n_{1}, \ldots, n_{i-1}, n_{i}+1, n_{i+1}, \ldots, n_{j-1}, n_{j}-1, n_{j+1}, \ldots, n_{k}\right), & \text { if } i<j \\ \left(n_{1}, \ldots, n_{j-1}, n_{j}-1, n_{j+1}, \ldots, n_{i-1}, n_{i}+1, n_{i+1}, \ldots, n_{k}\right), & \text { if } i>j\end{cases}
$$

$$
\begin{aligned}
& P_{\text {preempted }}(i)=\frac{N_{\text {preempted }}(i)}{N_{\text {accepted }}(i)} \\
& =\sum_{j=1}^{K} \sum_{n \in\left\{\left(n_{1}, \ldots, n_{K}\right) \mid n_{j}<r_{j}, i=\min \left\{h \mid n_{h}>r_{h}\right\}, \sum_{h=1}^{K} n_{h}=m\right\}} p(\bar{n}) \times \frac{\lambda_{j}}{\lambda_{i}} \times \frac{1}{1-P_{\text {block }}(i)}
\end{aligned}
$$




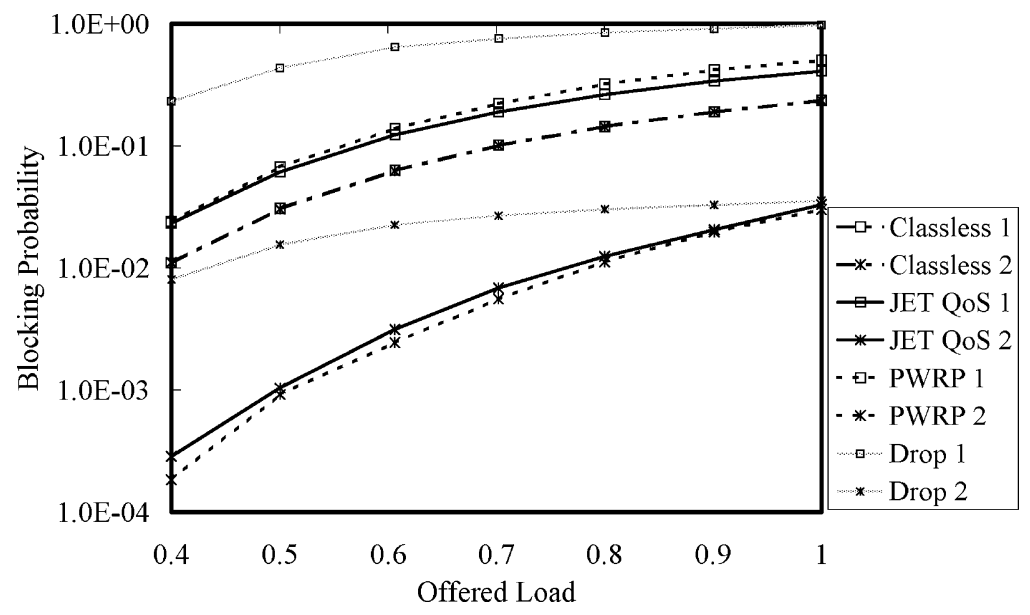

Fig. 4. Per-class blocking probabilities at a single switch.

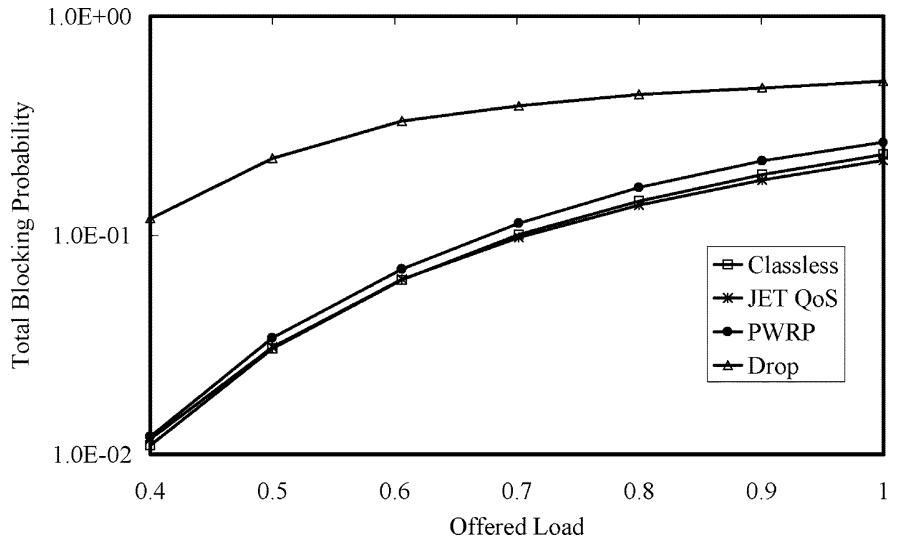

Fig. 5. Total blocking probabilities at a single switch.

rates. The blocking probabilities increase as the offered load increases for the four approaches. We see that that JET QoS, Drop, and PWRP all provide service differentiation for both classes of traffic. Compared with Drop, JET QoS and PWRP have lower blocking probabilities for the two classes of traffic, especially when the offered load is low. Fig. 5 shows the total blocking probabilities of the four approaches. The total blocking probabilities of both JET QoS and PWRP are very close to the classless case, while Drop has relatively higher blocking probability. Fig. 6 shows the system utilization for the four mechanisms. PWRP has the highest utilization (very close to the classless one), followed by JET QoS, and then Drop. The high utilization of PWRP is due to its ability to accumulate truncated bursts. JET QoS does not behave well under heavy load because high-priority traffic (i.e., class 2 ) has more opportunities to make reservations and may leave many gaps useless to low-priority bursts (e.g., too small to fit in). Thus, the utilization is low when the offered load is high. Drop behaves worse in a heavily loaded system due to intentional dropping.

To better observe the behavior of JET QoS under heavy load, we conduct another simulation to compare the proportional usage of each class in a heavily loaded system. Fig. 7 shows the proportional usage of the two classes of traffic for JET QoS and PWRP, varying the offered load from 0.7 to 2.7. In this experiment, the isolation factor of JET QoS is set to 0.6321

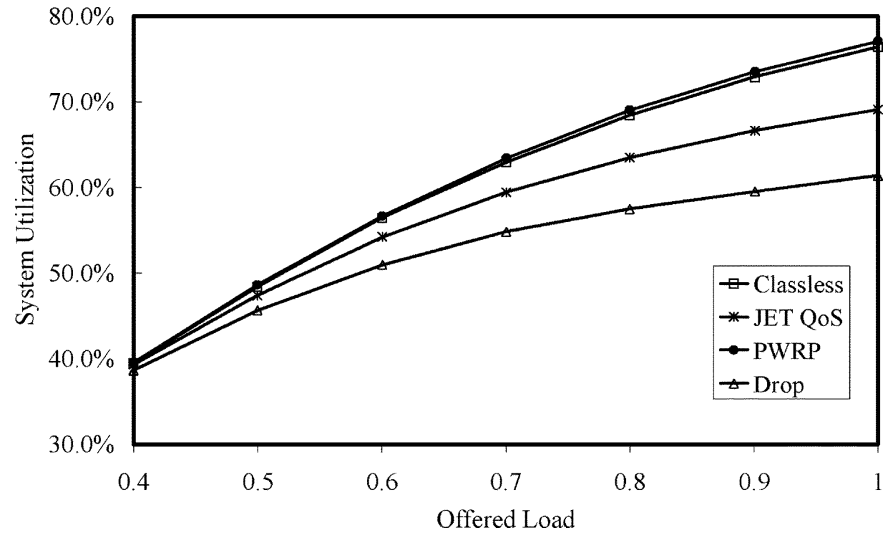

Fig. 6. System utilization at a single switch.

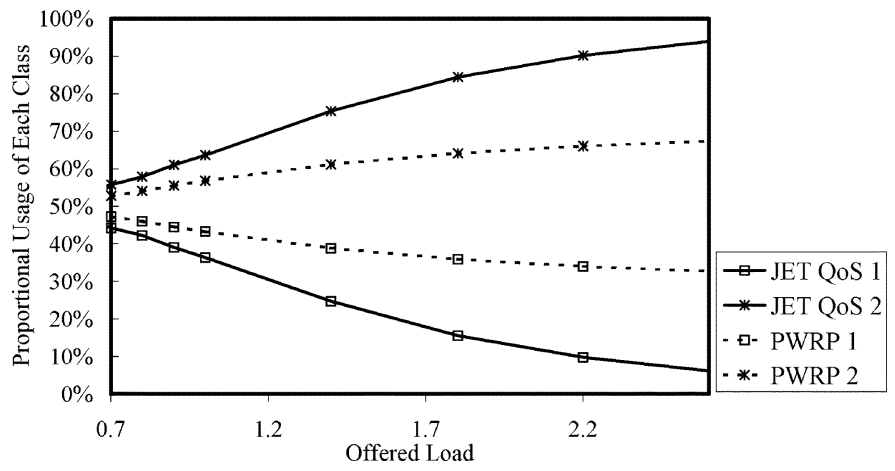

Fig. 7. Proportional usage under heavy load.

(i.e., offset time between classes 1 and 2 is set to one unit of average burst length). As described in [6], class 2 traffic will have a probability of 0.6321 to make reservation successfully if the isolation factor is set to 0.6321 . To have a fair comparison, the usage limits of classes 1 and 2 in PWRP are set to 0.3679 and 0.6321 , respectively. Again, there are eight outgoing wavelengths per output link at a bufferless switch. We see that under heavy load, class 2 of JET QoS obtains most of the resources, while class 1 of JET QoS only obtains a very small percentage of resources. Our approach always allocates resources to each class proportional to the predefined usage limit irrespective of whether the system is heavily loaded. Thus, the low-priority 


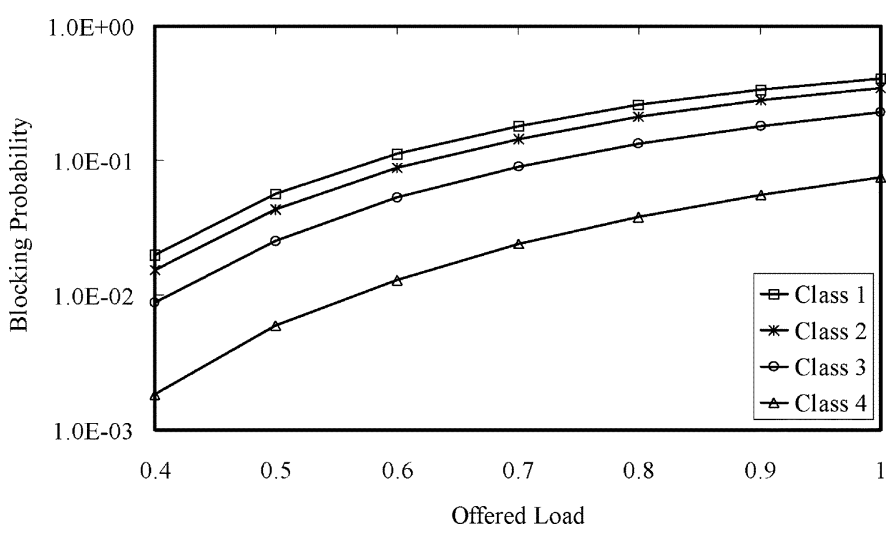

Fig. 8. Per-class blocking probabilities for multiple classes.

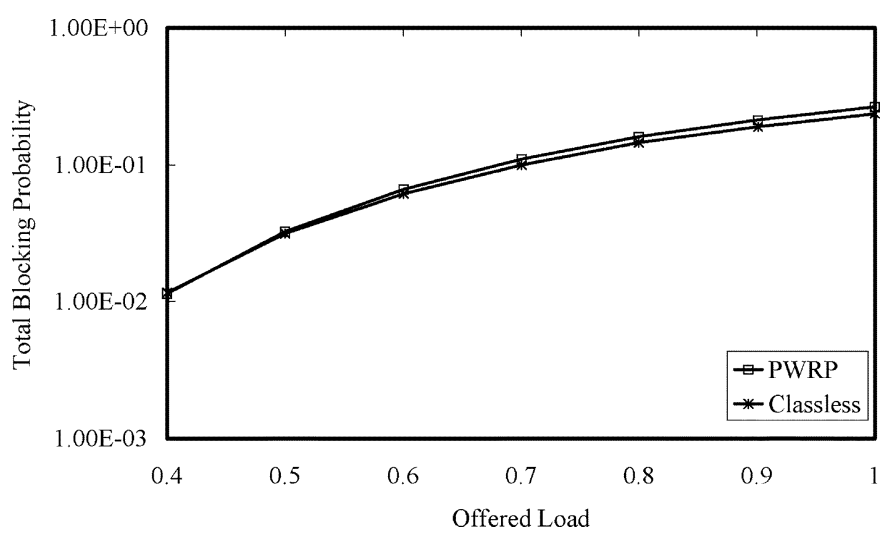

Fig. 9. Total blocking probability for multiple classes.

classes of traffic may be starved in JET QoS under a heavily loaded system, while our mechanism behaves fairly at all times.

We then extend the number of service classes from two to four with the following priorities: class $4>$ class $3>$ class $2>$ class 1 . Fig. 8 shows the class blocking probabilities of classes $1-4$, with usage limits of $0.75,0.2,0.05$, and 0.0 assigned to each of the four classes, respectively. The higher the offered load, the higher the blocking probability; the higher the priority, the lower the blocking probability. Fig. 9 shows the total blocking probability of PWRP in the multiclass case compared with the classless one. The performance of the two cases is very similar. Note that in this paper, we do not discuss how the usage limit of each class is assigned. For per-class blocking probability and usage limit, either can be derived by a priority queuing system with preemption when the other is given. Thus, the service provider can assign a usage limit to a class once the guaranteed blocking probability of the class is determined.

The next experiment is performed to observe how close the actual usage is to the usage limit of each class, varying the offered load from 0.4 to 1 . The usage limits of classes 1-4 are set to $0.12,0.13,0.25$, and 0.5 , respectively. The arrival rate and service rate of each class are set the same as in the first experiment. Since the traffic arrival rate of class 4 is always small as compared with its predefined usage 0.5 , the actual usage of class 4 is lower than its predefined usage limit. We observe how the other three classes of bursts contend for the extra resources released by class 4 . In this experiment, we compare two contention approaches. One is through free contention, which means the other

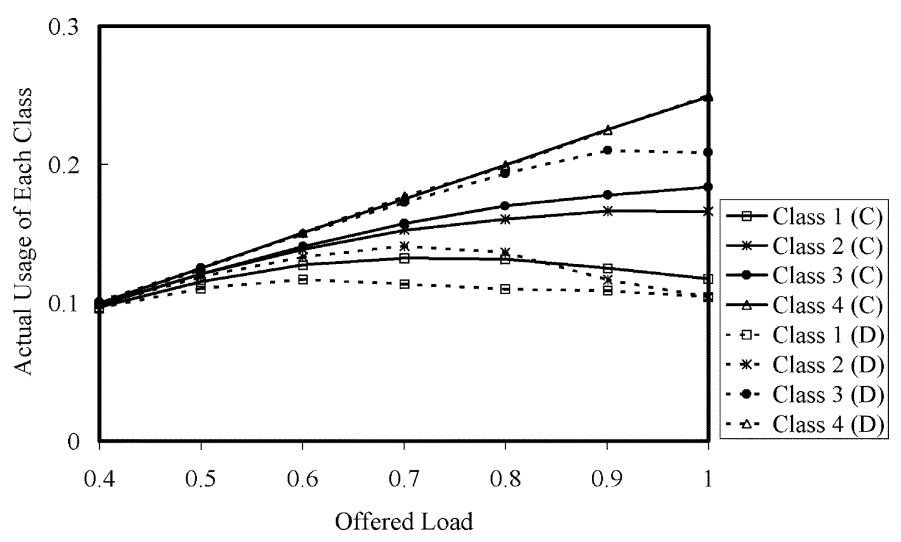

Fig. 10. Actual usage of each class.

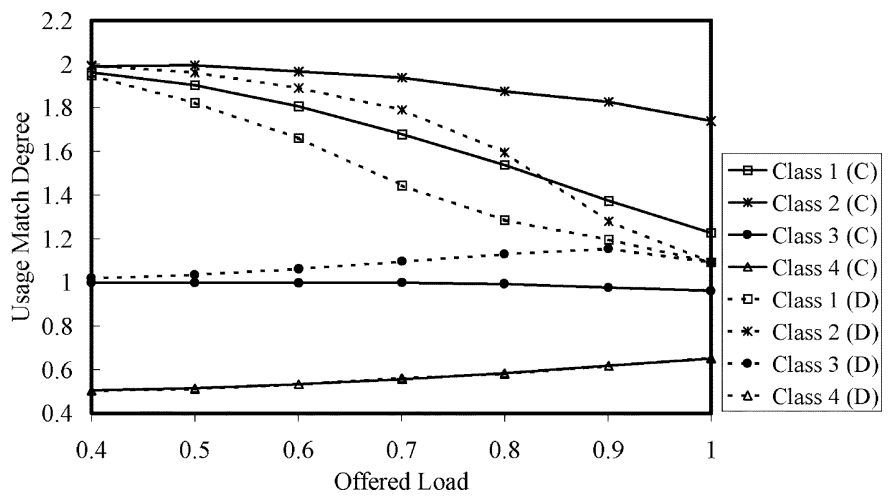

Fig. 11. Usage match degree of each class.

three classes of bursts freely contend for the residual resource. The other is through proportional distribution based on the corresponding service priority (i.e., the residual resource is proportionally distributed according to the normalized usage limit of each class).

Fig. 10 shows the actual resource usage of each class, and Fig. 11 shows the usage match degree of each class, all as a function of offered load. The usage match degree in the $y$ axis of Fig. 11 is defined as the ratio of the actual usage to the usage limit of each class. In both figures, the solid lines are for the free contention approach (indicated as $\mathrm{C}$ ), while the dashed lines are the proportional distribution (indicated as D). For both approaches, the match degree of class 4 is always below 0.7 due to its small arrival rate. The other three classes all have a match degree higher than one due to the extra resource released by class 4. We see that the proportional distribution approach uses resource more fairly, while the free contention one may reverse the service priority, i.e., lower priority bursts may use more resources than higher priority ones. (In this example, the solid line of class 2 is still lower than class 3 , but stays very close to each other.) For proportional distribution, the match degrees of all three classes reach one under heavy load. But when the offered load is low, low-priority classes tend to have high match degrees. This is because when the offered load is low, the predefined usage limits of high-priority classes are enough to accommodate their traffic, allowing the remaining resources to be distributed to the low-priority classes. Thus, the actual usages of low-priority classes are much higher than their predefined usage limits. This phenomenon can be observed in Fig. 11. The match 


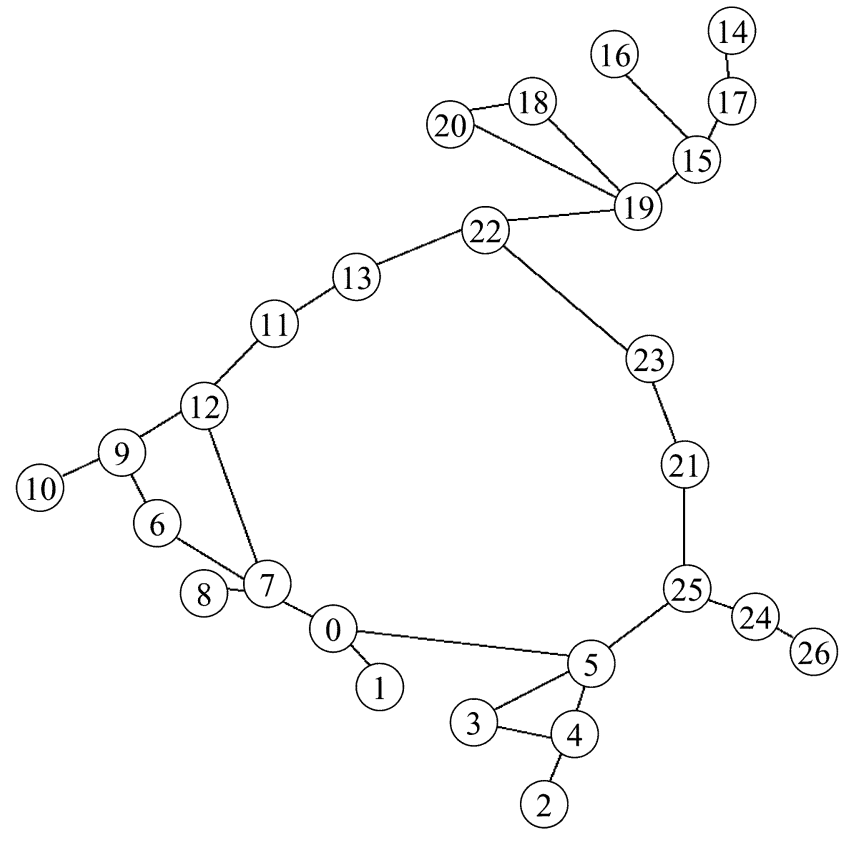

Fig. 12. A 27-node random topology network.

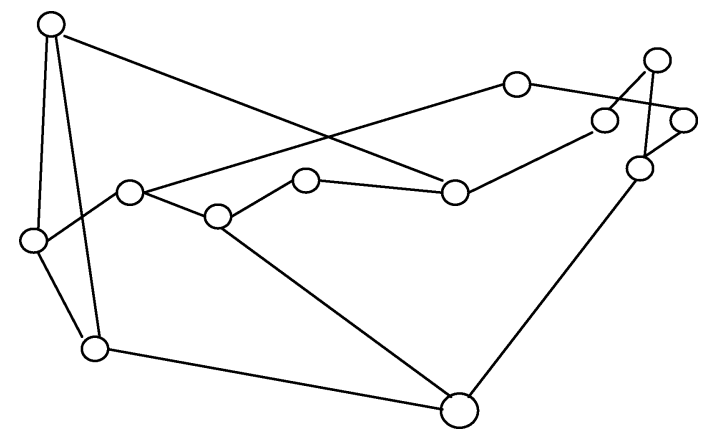

Fig. 13. A 13-node NSFNET.

degree of class 3 always remains at one under light load because its predefined usage limit is enough to serve its arrival rate. On the other hand, the match degrees of classes 1 and 2 are very high under light load due to the extra resources obtained from class 4 . However, when the system becomes more heavily loaded, class 3 bursts start contending for the extra resources with classes 1 and 2. Thus, the match degree of class 3 increases slightly, while the match degrees of classes 1 and 2 decrease.

In the next experiment, we extend from a single switch to a network of switches. We simulate two network topologies: one is a 27-node network with a hierarchical random topology generated by the GT-ITM generator (as shown in Fig. 12), and the other is a 13-node NSFNET (as shown in Fig. 13). The cost of each link in the figures is proportional to its length in the topology diagram. In this experiment, traffic is generated at edge routers. We also consider two classes of traffic, and the traffic model is the same as in the first experiment. Figs. 14 and 15 show the blocking probability of each class for the four mechanisms in both topologies. The results are consistent with the single-node case for both topologies, i.e., the three mechanisms all provide service differentiation for OBS networks, JET QoS

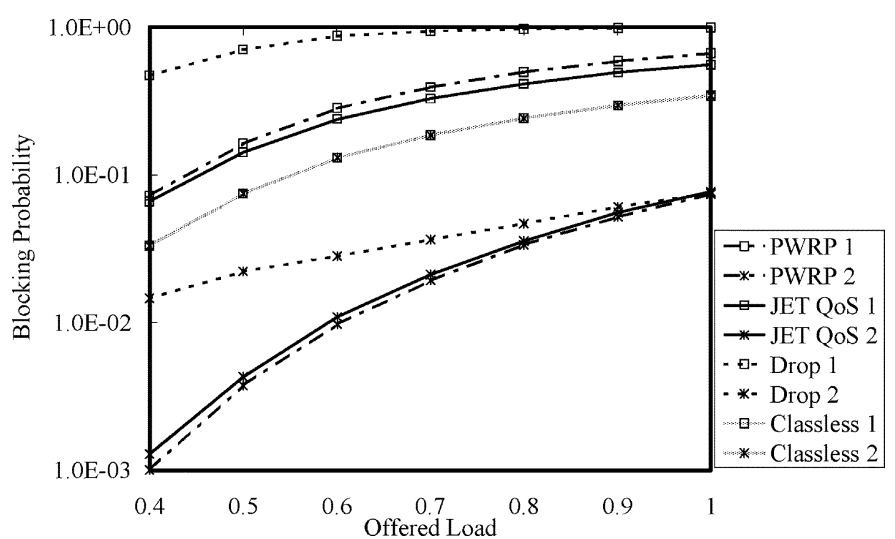

Fig. 14. Blocking probabilities for the random topology.

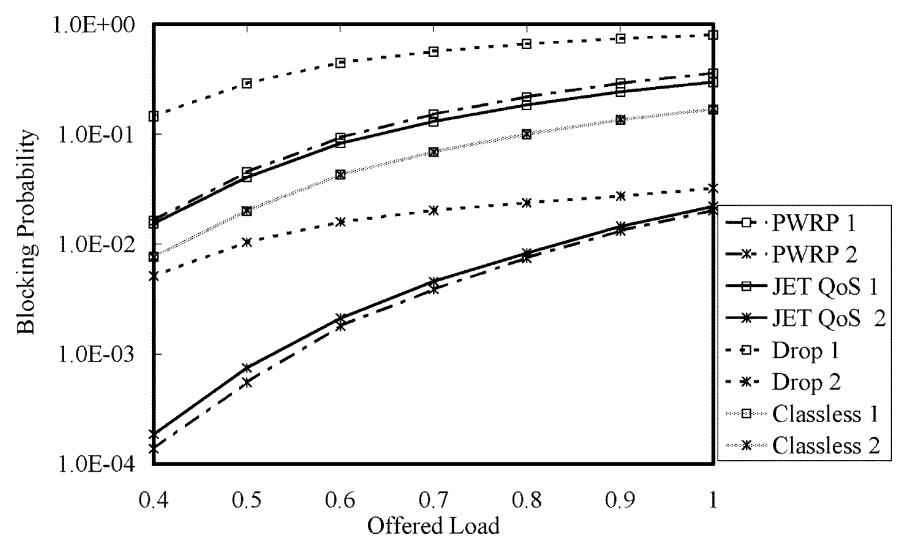

Fig. 15. Blocking probability for the NSFNET.

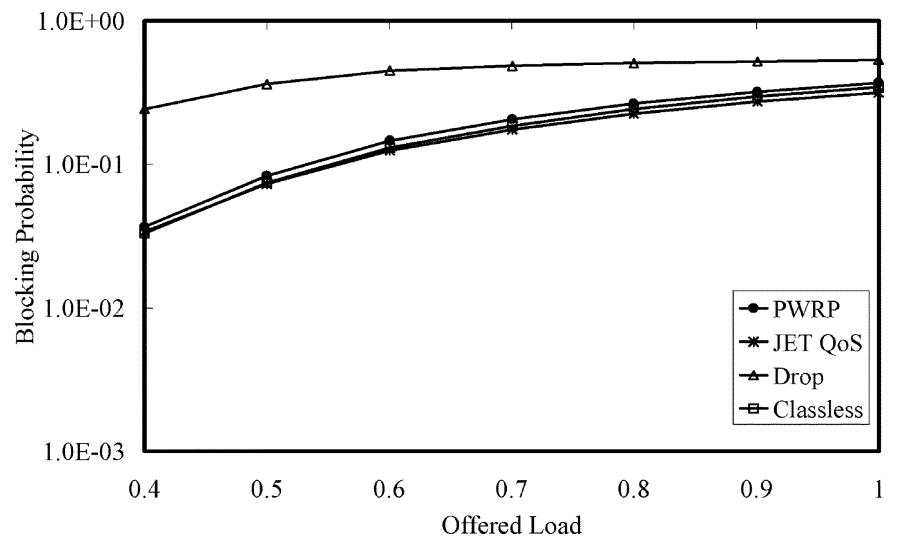

Fig. 16. Total blocking probability for the random topology.

and PWRP both have similar blocking probabilities for both classes, and Drop has higher blocking probabilities.

Fig. 16 shows the total blocking probabilities of the four mechanisms in the random topology network. ${ }^{2}$ Fig. 17 shows the system utilization of the four mechanisms. Again, Drop has the highest total blocking probability and the lowest system utilization, while the other three give similar performance. Note that the utilization of PWRP is slightly higher than that of classless due to the ability to accumulate truncated bursts. Drop has better utilization under light load. This is because the loss

\footnotetext{
${ }^{2}$ The results for the random topology and the NSFNET are very similar. To save space, we show only the simulation results for the random topology.
} 


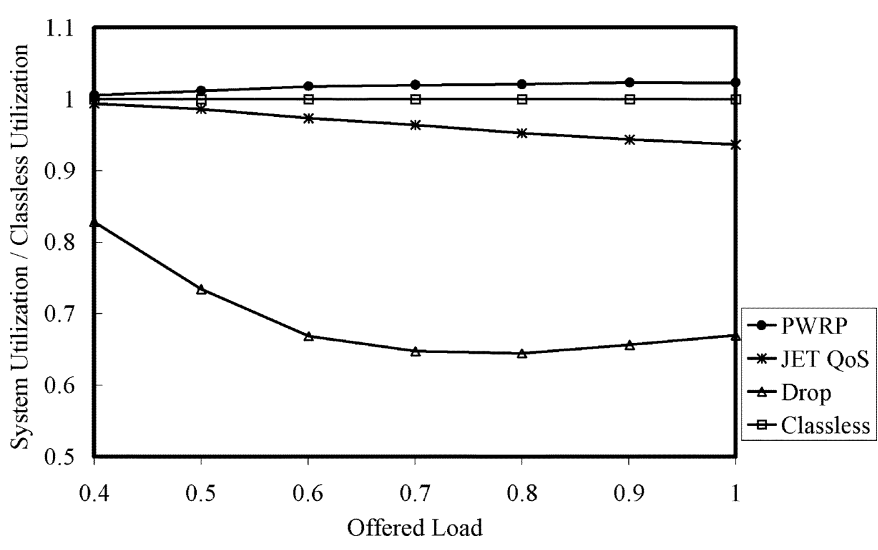

Fig. 17. System utilization for the random topology.

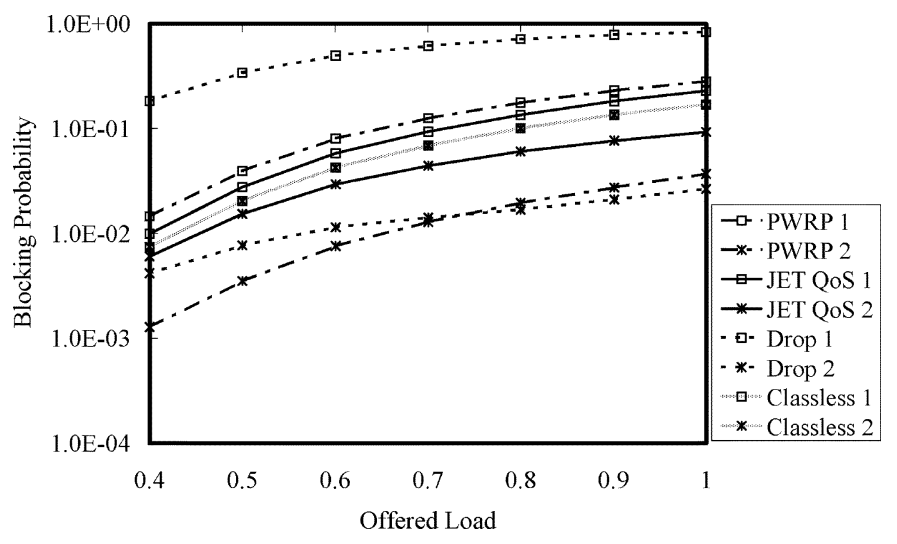

Fig. 18. Per-class blocking probabilities under congestion.

rate of each class is very low in the lightly loaded system. As a result, it is easy to satisfy the proportional factor under light intentional dropping. Therefore, its utilization is high if only little intentional dropping occurs.

The next experiment is to observe the performance of the four mechanisms under network congestion. The simulation is conducted in the 27-node random topology as shown in Fig. 12. Fig. 18 shows the per-class blocking probabilities of the four approaches when the offset delay time becomes invalid due to network congestion. It can be observed that both PWRP and Drop still provide differentiated service for classes 1 and 2, while JET QoS degrades into a classless scheme. Besides, Drop still has higher blocking probability than PWRP. Fig. 19 shows the system utilization of the four mechanisms under network congestion. Again, it is consistent with the noncongestion case (i.e., Fig. 17).

Thus, based on the simulation results, PWRP performs the best in terms of lower blocking probability and higher system utilization and is robust even under network congestion.

\section{Analysis Versus Simulation}

In this section, the analytical results are compared with the simulation. We consider four classes, i.e., class 1 , class 2 , class 3 , and class 4 , with ascending priorities. The usage limits of classes $4-1$ are set to $0.5,0.25,0.125$, and 0.125 , respectively. In other words, the wavelengths used by each class at time $t$ are $4,2,1$, and 1, respectively. Fig. 20 shows the blocking probabilities of new incoming bursts, and Fig. 21 depicts the preempted

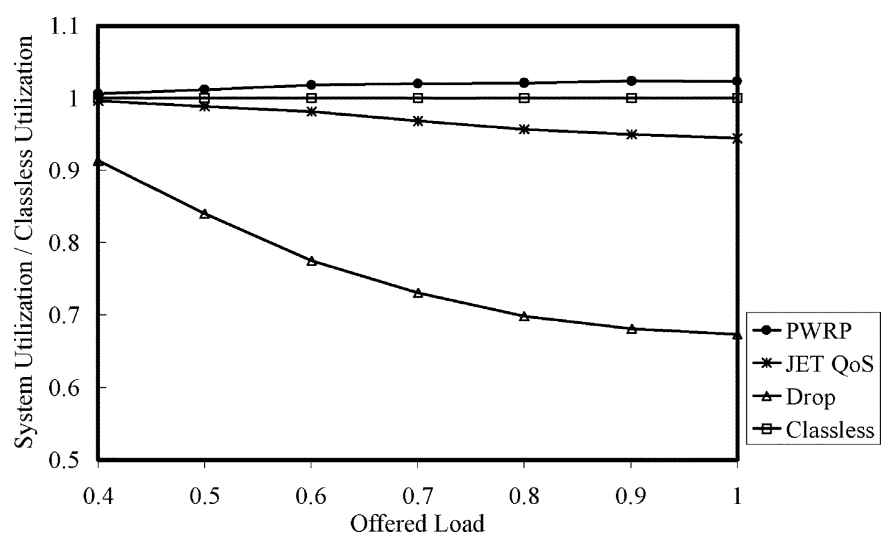

Fig. 19. System utilization under congestion.

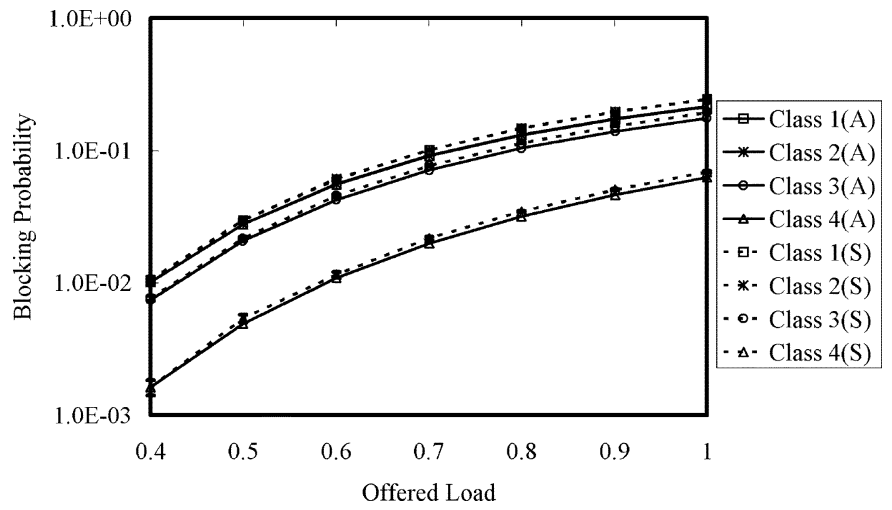

Fig. 20. Blocking probabilities of new bursts.

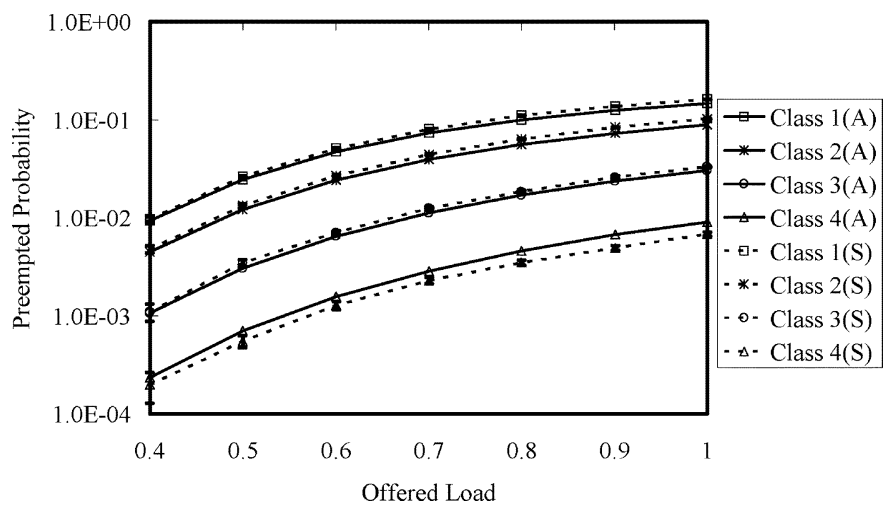

Fig. 21. Preempted probability of an accepted call.

probabilities of accepted bursts. In both figures, the solid lines (indicated by A) are from the analytical results, and the dashed lines (indicated by S) are from the simulation. The $98 \%$ confidence interval of the simulation values are within \pm 0.001 of the mean values shown. The analytical results match the simulation results very well. Note that since the blocking probabilities of classes 1 and 2 are very similar, the curves of both classes in Fig. 20 overlap.

\section{CONCLUDING REMARKS}

In this paper, a new bufferless mechanism called PWRP is proposed to provide a preemptive wavelength reservation mechanism for service differentiation in OBS networks. Unlike 
existing approaches, which may degrade to classless schemes (e.g., JET QoS) or which may suffer from low wavelength utilization (e.g., Drop), the proposed mechanism is robust, efficient, and supports an incremental deployment of QoS support. In PWRP, a usage profile is maintained for each class at the router, and a preemptive wavelength reservation mechanism is implemented to ensure QoS. An analytical model is derived and simulations conducted to evaluate the performance. From the simulation and analytical results, PWRP outperforms JET QoS and Drop in terms of lower blocking probability and higher resource utilization, making the proposed approach an excellent QoS mechanism for OBS networks.

\section{REFERENCES}

[1] J. Turner, "Terabit burst switching," J. High Speed Networks, vol. 8, pp. 3-16, 1999.

[2] C. Qiao and M. Yoo, "Optical burst switching (OBS) - A new paradigm for an optical internet," J. High Speed Networks, vol. 8, no. 1, pp. 69-84, 1999.

[3] H. Zhang, "Service disciplines for guaranteed performance service in packet switching networks," Proc. IEEE, vol. 83, pp. 1374-1396, Oct. 1995.

[4] A. Varma and D. Stilladis, "Hardware implementation of fair queuing algorithms for asynchronous transfer mode networks," IEEE Commun. Mag., vol. 35, pp. 74-80, Dec. 1997.

[5] U. Briem et al., "The traffic management for an ATM switch with per-VC queuing: Concept and implementation," IEEE Commun. Mag., vol. 36, pp. 88-93, Jan. 1998.

[6] M. Yoo and C. Qiao, "Supporting multiple classes of services in IP over WDM networks," in Proc. IEEE GLOBECOM'99, 1999, pp. 1023-1027.

[7] Y. Chen, M. Hamdi, and D. H. K. Tsang, "Proportional QoS over OBS networks," in Proc. IEEE GLOBECOM 2001, vol. 3, Nov. 2001, pp. $1510-1514$.

[8] K. Dolzer, "Assured horizon-An efficient framework for service differentiation in optical burst switched networks," in Proc. SPIE/IEEE OPTICOMM 2002, July 2002, pp. 149-159.
[9] V. Vokkarane and J. Jue, "Prioritized burst segmentation and composite burst assembly techniques for QoS support in optical burst-switched networks," IEEE J. Select. Areas Commun., vol. 21, pp. 1198-1209, Sept. 2003.

[10] E. Kozlovski, M. Duser, A. Zapata, and P. Bayvel, "Service differentiation in wavelength-routed optical burst-switched networks," in Proc. IEEE Optical Fiber Communications (OFC'02), Mar. 2002, pp. 774-776.

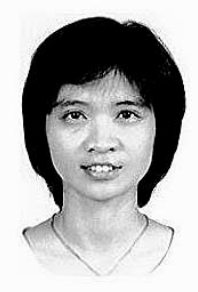

Wanjiun Liao (S'96-M'97) received the B.S. and M.S. degrees from National Chiao Tung University, Taiwan, R.O.C., in 1990 and 1992, respectively, and the $\mathrm{Ph} . \mathrm{D}$. degree in electrical engineering from the University of Southern California, Los Angeles, in 1997.

She joined the Department of Electrical Engineering, National Taiwan University (NTU), Taipei, Taiwan, R.O.C., as an Assistant Professor in 1997. Since August 2000, she has been an Associate Professor. Her research interests include wireless networks, overlay networks, and Internet protocols.

Dr. Liao has received many research awards, including the Outstanding Research Paper Award in Electrical Engineering at the University of Southern California in 1997 and the Best Student Paper Award at the First IEEE International Conferences on Multimedia and Expo (ICME) in 2000 and the Best Paper Award at the First International Conference on Communication, Circuits and Systems (IEEE Communication Press) in 2002, two papers she coauthored with her students. She was elected as one of the Ten Distinguished Young Women in Taiwan in 2000 and is listed in the Marquis Who's Who in 2001-2003 and the Contemporary Who's Whoin 2003. She is currently an Associate Editor for IEEE TRANSACTIONS ON WIRELESS COMMUNICATIONS.

Chi-Hong Loi received the B.S. and M.S. degrees in electrical engineering from National Taiwan University, Taiwan, R.O.C., in 2000 and 2002, respectively. $\mathrm{He}$ is now an Engineer in SENAO International Company, Taiwan, R.O.C. 